\title{
Toward V2I Communication Technology-based Solution for Reducing Road Traffic Congestion in Smart Cities
}

\author{
Soufiene Djahel ${ }^{\mp}$, Nafaa Jabeur ${ }^{\ddagger}$, Robert Barrett ${ }^{\mp}$ and John Murphy ${ }^{\mp}$ \\ ₹ UCD School of Computer Science and Informatics, Ireland \\ $¥$ German University of Technology in Oman (GUtech), Sultanate of Oman \\ \{soufiene.djahel, j.murphy\}@ucd.ie, nafaa.jabeur@gutech.edu.om, robert.barrett.1@ucdconnect.ie
}

\begin{abstract}
Due to the limited capacity of road networks and sporadic on-route events, road traffic congestions are posing serious problems in most big cities worldwide and resulting in considerable number of casualties and financial losses. In order to deal efficiently with these problems and alleviate their impact on individuals, environment, and economic activities, advanced traffic monitoring and control tools (e.g., SCATS and SCOOT) are being widely used in hundreds of major cities in the world. However, due to increasing road traffic and dynamic spatiotemporal events, additional proactive mechanisms remain needed to prevent traffic congestions. Within this context, we argue that the emergent V2X communication technologies, and especially V2I (Vehicle to Infrastructure), would be of great help. To this end, we investigate in this paper the opportunities that could be offered by V2I technology in improving commuters' journey duration and mitigating the above irritating and frequent problems. We then propose an approach where road-side facilities (e.g. traffic light controllers at road intersections) communicate traffic light cycle information to approaching vehicles. Based on this information, the vehicles collaboratively determine their optimal speeds and other appropriate actions to undertake in order to cross road intersections with minimum delays while ultimately avoiding stoppings. The obtained evaluation results show that our approach achieves a significant gain in terms of the commuters' average travel time reduction.
\end{abstract}

Keywords - Vehicular Communication, V2I, V2V, Traffic Congestion, Smart Cities.

\section{INTRODUCTION}

Nowadays, most of growing cities in the world are witnessing an unprecedented increase in road traffic congestion due to the fast growing world-wide urbanization. Traffic congestion is, therefore, regarded as one of the main challenges being faced by traffic management authorities and experts worldwide. While recurrent congestions (e.g. large number of vehicles simultaneously using a limited road section during peak day hours) are relatively easy to predict and could be solved with planned actions, non-recurrent congestions (e.g., randomly crashed or stalled vehicles, work zones, bad weather conditions, etc.) are hard to anticipate, making thereby the design of appropriate and timely response plans a real challenge. Traffic congestions generally result in substantial economic losses, including increased fuel consumption, productivity troubles (because of employees' absenteeism and lateness), and delivery disruption. In addition to human live losses [2], [3], statistics published in [1] reveal that the incurred economic loss in the U.S. is estimated as $\$ 121$ billion in 2011 and is expected to increase up to $\$ 199$ billion in 2020. Because of these huge losses, decision-makers are being pressurized to bring solutions to traffic congestion problems and particularly remediate appropriately and on-time to sporadic unplanned events.

Several commercial solutions (e.g., from Garmin and TomTom) are already available. Devices proposed by these solutions are essentially based on Global Positioning System (GPS) technologies. They receive and analyze frequent updates from traffic reporting systems, monitoring devices, and/or dedicated equipment (e.g., plate number recognition systems). Alternative routes could then be suggested based on the results of the analysis. Current commercial solutions, however, do not implement any mechanism allowing devices installed in vehicles to communicate with the road network infrastructure controllers or to communicate between each other.

The academic community has also investigated the road traffic congestion problem and designed several solutions to assist the Traffic Management System (TMS) in dealing efficiently with the aforementioned issues. These solutions have spanned the whole life cycle of traffic data in smart transportation domain, ranging from advanced data collection devices and protocols using WSNs (Wireless Sensor Networks) technology (e.g., [4], [9] and [18]) to route planning (e.g., [5], [6]) and short term traffic prediction techniques (e.g., [7], [8]). Recently, researchers from academia have also designed futuristic approaches and visions of adaptive TMS based on V2X communication to ensure secure and efficient emergency and non-emergency service delivery (e.g., [11], [13] and [12]). Besides, vehicle manufacturers such as General Motors, Toyota and Nissan, among others, are also actively promoting the development of $\mathrm{V} 2 \mathrm{~V}$ (Vehicle to Vehicle) communication-based solutions and integrating the required equipment into their vehicles to support both safety and non-safety applications.

In order to contribute to the aforementioned ongoing efforts and provide current solutions with additional mechanisms capable of reducing the vehicles waiting delay at intersections, we propose to leverage the communication between vehicles and Traffic Lights Controllers (TLCs) as well as between vehicles themselves. To this end, vehicles are, first, fed with 
information from the TLC as well as with their mutual data. They, then, collaborate with the aim to coordinate their speeds and actions (e.g., lane change) to finally reduce waiting times in road intersections. The collaboration also aims to avoid stoppings when and where this is possible.

The remainder of the paper is organized as follows. Section II presents the basic ideas and concepts of our solution along with its detailed operation. Section III evaluates the performance of the proposed solution and analyzes the obtained simulation results. Finally, we conclude in Section IV.

\section{PROPOSED SOLUTION}

\section{A. Key idea}

Our ultimate goal in this paper is to reduce traffic congestion while avoiding, whenever and wherever possible, stoppings. We argue that this is possible through an improved coordinated and controlled acceleration/deceleration of vehicles and an increased awareness of their surroundings. To meet this goal, we propose an approach based on priority mechanisms to manage Vehicle-to-Infrastructure (V2I) and Vehicle-to-Vehicle (V2V) communications. Within this context, each TLC broadcasts periodically its status data to the vehicles running on the road segments that it controls, as illustrated in Figure 1. Afterwards, these data will be processed by the receiver vehicles and appropriate adjustment to their speed as well as other actions will be taken in coordination with their neighbors for optimal efficiency. Vehicles should not be selfish in their actions. Each vehicle (ultimately the driver) should, indeed, adjust its acceleration, deceleration, and even change lane to accommodate as many other vehicles around to cross the road intersection as possible. Taken collaboratively, these actions are based on information received from the TLC as well as from mutual exchange of periodic beacons ${ }^{1}$. In order to improve the decision-making process during their collaboration, vehicles could also identify and exchange information about selfishly acting vehicles by piggybacking such information into the standard beacon message.

In addition to the beacons generated by individual vehicles, the TLCs also generate and broadcast periodic beacons particularly containing information on current traffic controllers' states (green, amber, or red) as well as the remaining time (in seconds) before these states change. The transmission period of these beacons is well tuned and adapted to the road segment length and average speed of vehicles such that each vehicle will receive at least one beacon before it reaches the intersection. It is worth to mention that, in this paper, we neglect the change to the amber state as it is merely used to attract the attention of drivers about an imminent state change. Moreover, to ensure reliable transmission of the beacons transmitted by both vehicles and TLCs a congestion control mechanism could be used such as the approach proposed in [17].

\footnotetext{
${ }^{1}$ The beacons exchanged between vehicles are transmitted periodically at least 10 times per second.
}

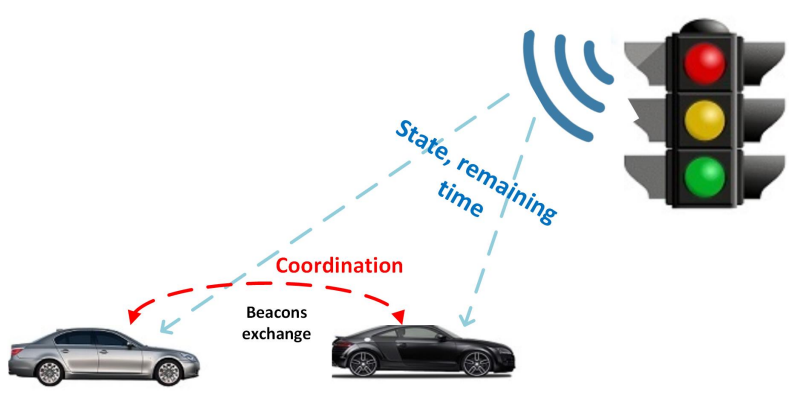

Figure 1: TLC-vehicles interaction

\section{B. Belief-Desire-Intension architecture for vehicles}

In order to allow the vehicles to reason adequately about occurring events and the dynamic configuration within the surrounding environment, we propose a Belief-Desire-Intension (BDI) architecture [10] for every vehicle as depicted in Figure 2. In this architecture, beliefs represent the local information that the vehicle has about itself (e.g., its current speed, position, remaining distance to the intersection) and the environment (including the road infrastructure, neighboring vehicles, and events of interest like announced obstacles/crashes ahead). Beliefs could be true or false and are subject to change. The desires reflect the objectives or the situations that the vehicle would like to accomplish, whereas the intentions refer to the actions that the vehicle has chosen to do. According to this architecture, a vehicle holds a set of knowledge about itself, the road traffic system, neighboring vehicles, and any event of interest in the vicinity (e.g., vehicle crash, water accumulated because of rain). The vehicle will be always listening to beacons and communications from neighboring vehicles as well as the TLC. Once new information are received, a revision function is executed in order to update the current beliefs. Based on the new beliefs, an option generation function updates the desires of the vehicle. An action generation function is then applied to deliberate the new intensions of the vehicle. A plan generation function is finally executed to schedule the actions of the vehicle and update the beliefs, desires, and intentions accordingly.

\section{Detailed operation}

The proposed approach is performed over several steps, as illustrated in Figure 3. Every step results in a set of recommendations to each individual vehicle about the appropriate actions to carry out, such as changing lane and decelerating or accelerating without exceeding the legal speed limit on the road network (i.e. the current road segment). These recommendations are inferred based on in-vehicle available information, from both VNS (Vehicle Navigation System) and local sensors as well as information collected from the received beacons. Moreover, for the purpose of achieving cost effective computation, the information analyzed by each vehicle are limited to those relevant to the current situation and decision to be taken. 


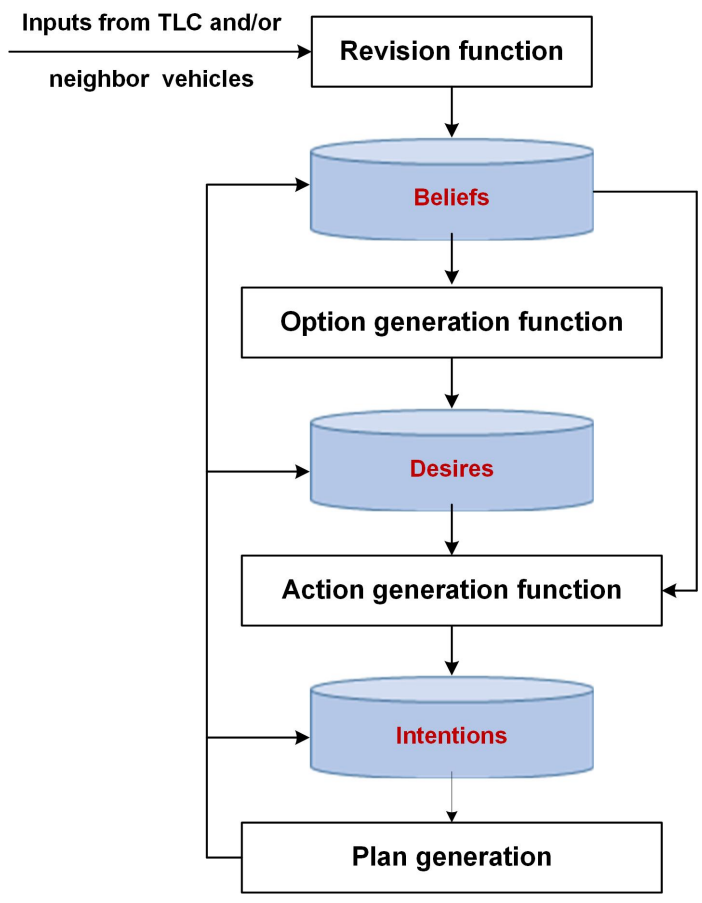

Figure 2: Belief-Desire-Intention architecture of vehicles

Upon reception of a beacon message, the vehicle will proceed as follows (see Figure 3): it first updates its beliefs about the environment and then checks its current situation to evaluate and assess its objectives, which could be, in this scenario, to cross the road intersection without stopping and serving other vehicles if the situation and speed limit allow (e.g., accelerate to allow other vehicles behind to cross the intersection, assist other vehicles to change lanes, etc.). Based on the new objectives, the vehicle will decide to accelerate, decelerate, keep the same speed, or change lane while coordinating these intentions with the other neighboring vehicles for safety and efficiency purpose. When the vehicle decides to accelerate, specific mechanisms are used in order to determine the optimal speed using information about the distance to the road intersection, remaining time to change the status of the traffic lights, the speed of the vehicles ahead, and the legal speed limit. Moreover, the safety distance between the vehicle and its neighboring vehicles ahead and behind should be maintained. Any change that occurs within a given step is stored locally at the vehicle and advertised in the next beacon.

During critical moments resulting from vehicles' decisions and actions as well as sudden contextual events, an immediate beacon transmission could be triggered during either CCHI (Control Channel Interval) or SCHI (Service Channel Interval). If the beacon transmission is initiated during the SCHI then this beacon will be encapsulated in a regular service packet and retransmitted again during the next CCHI. However, if we assume that all vehicles are equipped with multi radio devices then the above beacon can always be transmitted over the $\mathrm{CCH}$ since in this scenario no alternation between $\mathrm{CCH}$ and $\mathrm{SCH}$ is needed. In our solution, this beacon has twofold objective as described below. It may implicitly request an immediate cooperation from the neighboring vehicles in order to either facilitate the sender vehicle's lane change action (i.e. ensure that it will be fast and safe) or accommodate surrounding accelerating and decelerating vehicles while maintaining safety distances. The beacon may also aim to inform the vehicle(s) behind the sender vehicle about its acceleration in order to allow them to cross the intersection prior to the traffic lights state change.

\section{Beacons format}

In order to efficiently implement our solution we use the standard IEEE $802.11 \mathrm{p}$ developed specifically to support Intelligent Transportation System (ITS) applications by adapting and extending IEEE802.11a to enable Wireless Access in Vehicular Environments (WAVE) [14]. This standard is particularly chosen because of its high popularity and wide use in US and EU as a vehicle information transmission protocol. Communication in the real world is performed in the licensed frequency band of $5.9 \mathrm{GHz}$.

Apart from the standard header used in IEEE802.11 MAC frame, for the specific needs of our solution, we dedicate a portion of the available 2312-bytes that can be used in the data field for our beacons payload as follows: 764-bit for the TLCs beacon and 716-bit for the vehicles beacon. Since the authentication and data confidentiality (security) mechanisms provided by the IEEE 802.11 standard are not used in IEEE $802.11 \mathrm{p}$, they can also be defined in the payload, if necessary. Figure 4 depicts an example of two wireless frames representing the beacons broadcasted by individual vehicles as well as by the TLC. The first four bits in both frames are dedicated to the entity type. These bits will allow the receiver vehicle to identify the type of the sender entity, namely vehicle or TLC. Notice here that the additional 3 bits in this field are used to accommodate the identification of special categories of vehicles which may require particular actions/decisions to be taken such as ambulances and police cars.

The rest of the bits in both payloads hold several important information, including Vehicle Identification Number (VIN), the current speed of vehicle, the current vehicle signals (drivers' intentions to change lane, braking, etc.), its position in a given lane, and the distance separating it to the vehicle in front. The bits also hold information on events ahead, which could be used to relay information to the vehicles about either unplanned delays (such as vehicle collisions or accidents) or planned delays (such as concerts or sporting events) ahead. This could then be used, for example, by vehicles for better rerouting decisions.

\section{Performance evaluation}

\section{A. Simulation Setup}

Initial testing was performed using the GUI (Graphical User Interface) of SUMO [16] as well as its TraCI extension [15]. Eclipse was used as the IDE along with its Python language 


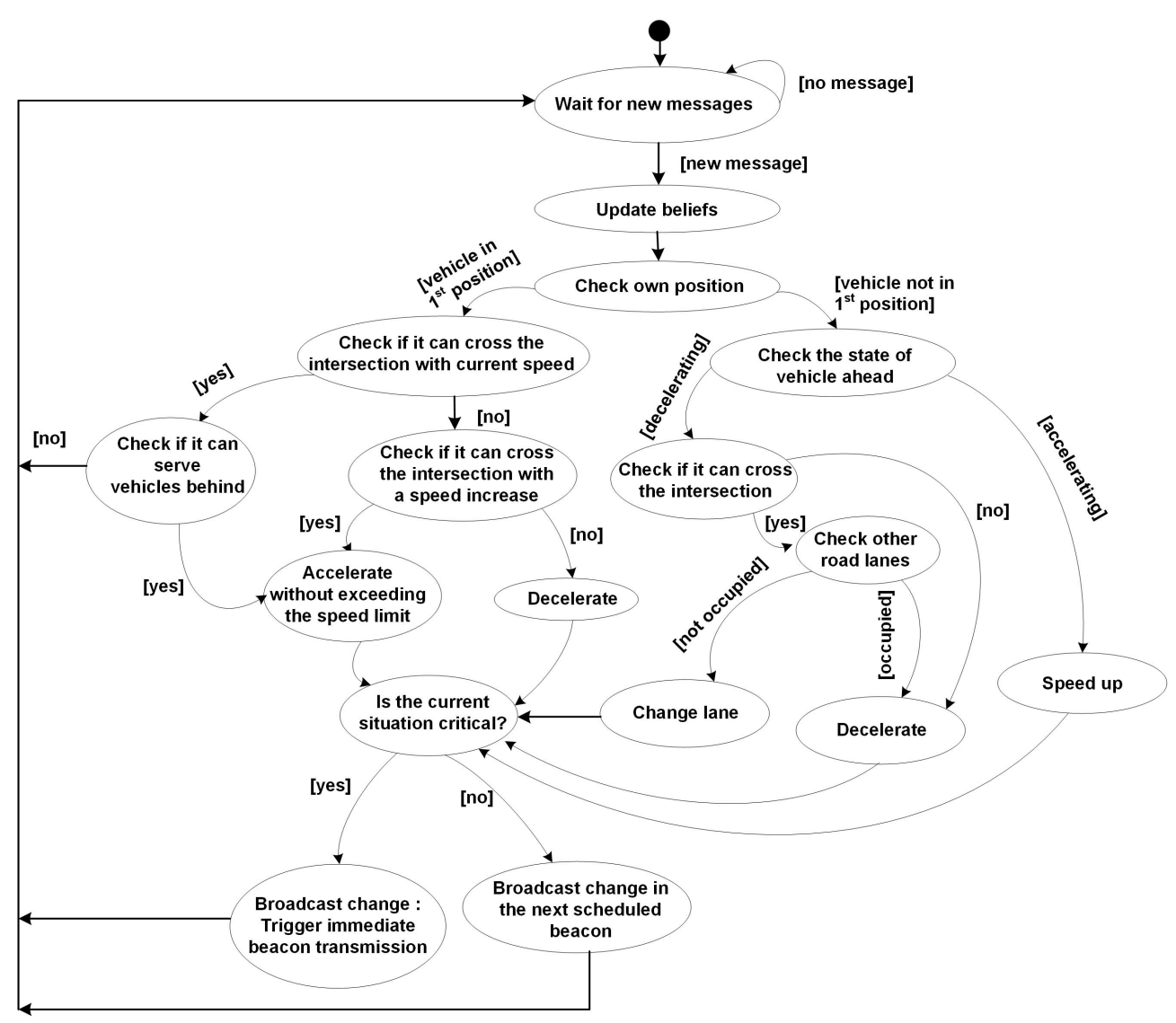

Figure 3: Detailed operations of each vehicle

\begin{tabular}{|c|c|c|c|c|}
\hline $\begin{array}{c}\text { Entity Type } \\
\text { (4 bits) }\end{array}$ & $\begin{array}{c}\text { Traffic Controller ID } \\
\text { (136 bits) }\end{array}$ & $\begin{array}{c}\text { Current Traffic Lights State } \\
\text { (48 bits) }\end{array}$ & $\begin{array}{c}\text { Time Before State Change } \\
\text { (64 bits) }\end{array}$ & $\begin{array}{c}\text { Incidents Ahead } \\
\text { (512 bits) }\end{array}$ \\
\hline
\end{tabular}

A. Traffic Lights Controller Beacon Format

\begin{tabular}{|c|c|c|c|c|c|c|c|c|c|c|}
\hline $\begin{array}{c}\text { Entity Type } \\
\text { (4 bits) }\end{array}$ & $\begin{array}{c}\text { VIN } \\
\text { (136 bits) }\end{array}$ & $\begin{array}{l}\text { Road ID } \\
\text { (64 bits) }\end{array}$ & $\begin{array}{l}\text { Lane ID } \\
\text { (64 bits) }\end{array}$ & $\begin{array}{l}\text { Lane } \\
\text { Position } \\
\text { (64 bits) }\end{array}$ & $\begin{array}{c}\text { Acceleration } \\
\text { (64 bits) }\end{array}$ & $\begin{array}{l}\text { Current } \\
\text { Speed } \\
\text { (64 bits) }\end{array}$ & $\begin{array}{c}\text { Deceleration } \\
\text { (64 bits) }\end{array}$ & $\begin{array}{l}\text { Signals } \\
\text { (64 bits) }\end{array}$ & $\begin{array}{l}\text { Waiting Time } \\
\text { (64 bits) }\end{array}$ & $\begin{array}{c}\text { Gap } \\
\text { (64 bits) }\end{array}$ \\
\hline
\end{tabular}

Figure 4: The format of beacons sent by the TLC (A) and individual vehicles (B)

extensions. SUMO allows each vehicle to be modelled individually, whereas TraCI implements several mechanisms to control each of these vehicles during simulation. The implementation of our scheme consists in using the vehicle data and the TLC data to control vehicles and imitate drivers (or smart cars) responding to suggested changes in velocity or lanes. For the sake of illustration, and for an accurate reflection of how our proposed scheme would perform in a real life scenario, two maps were chosen. These maps (representing Bunker Hill in Los Angeles as shown in Figure 7, and Lower Manhattan, New York City (NYC), USA as depicted in Figure 8) were imported from OpenStreetMap using SUMOs NETCONVERT function. The areas covered by the maps are all 1.8 miles in height and 1.5 miles in width and the standard grid layout of SUMO was used as a control. Los Angeles and Manhattan differ somehow in their layouts, so they were ideal for simulating different urban roads configurations. Los Angeles tends to have rather wide roads, with ample two or more way systems, whereas New York tends to have narrower streets, a lot being one way systems. Most of big cities tend to lean on either side of this spectrum or somewhere in the middle of both. We thus believe that our simulation results on the two selected configurations should apply well to the other cities.

The performance of our scheme is evaluated using the above three maps with four different traffic loads (i.e., 120 vehicles per $\mathrm{km}^{2}, 240$ vehicles per $\mathrm{km}^{2}, 360$ vehicles per $\mathrm{km}^{2}$ and finally 480 vehicles per $\mathrm{km}^{2}$ ). Our scheme was also compared against another baseline scheme. In this latter, 


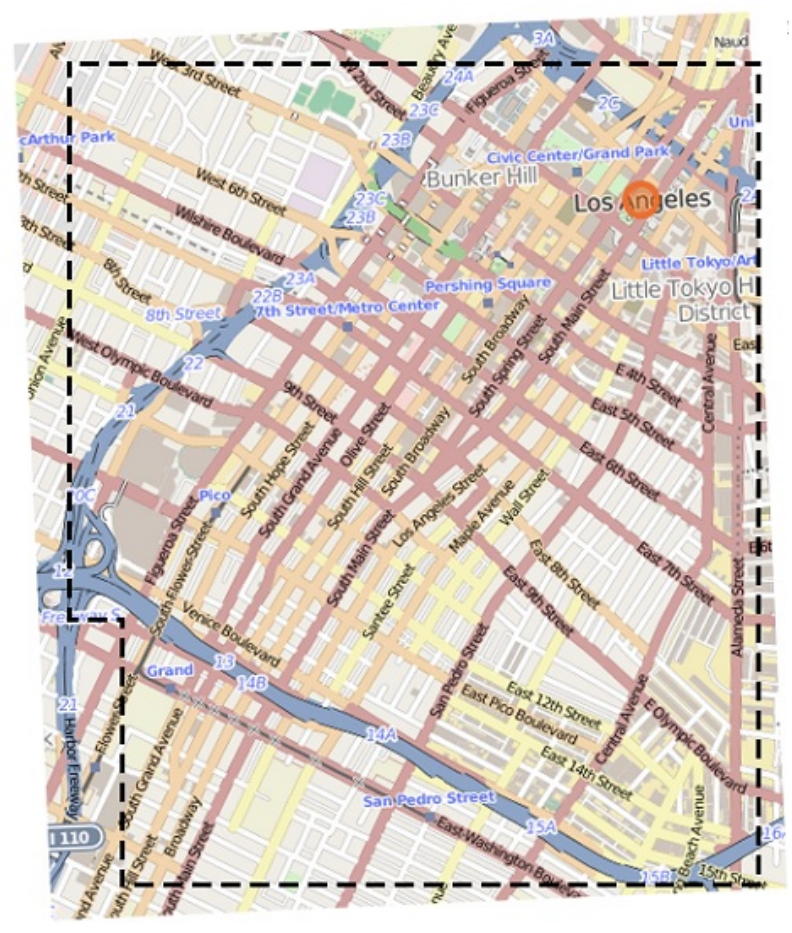

Figure 5: Bunker Hill map in Los Angeles

the vehicles exchange beacons and coordinate their actions to ensure safety and maintain security distances but no beacon is generated by the TLC (i.e., the TLC here operates as any regular traffic light controller used in current road networks). In our simulation, 120 vehicles per $\mathrm{km}^{2}$ represents light load on the road network, which reflects off-peak traffic conditions. Traffic flow is consistent at this level and stoppages are not a major problem, however because our proposed solution coordinates acceleration and deceleration actions of vehicles, we are expecting to see some impact on the achieved travel time. This impact is expected to increase with the density of vehicles. The simulations were run 10 times for each of the above vehicle density levels, and for each scenario.

\section{B. Simulation results}

In our simulation, vehicles density levels were gradually increased from light to higher loads and an upper limit of 3500 seconds per journey was applied, to remove outliers that would not benefit the accuracy of the data.

The performance evaluation metrics that have been measured during our simulation are: Average Travel Time (ATT), Traffic Load (TL) and the Coefficient of Variation (CV). The ATT represents the average time during which every vehicle (minus the major outliers as stated previously) has completed its journey. The TL is the current number of vehicles running on a given lane compared to its capacity (i.e. the number of vehicles that it can handle). This metric is mainly used to highlight the effect of our scheme on the traffic load balance that most of advanced TMSs aim to accomplish. Finally, the $\mathrm{CV}$ is the standard deviation in comparison to the ATT. This

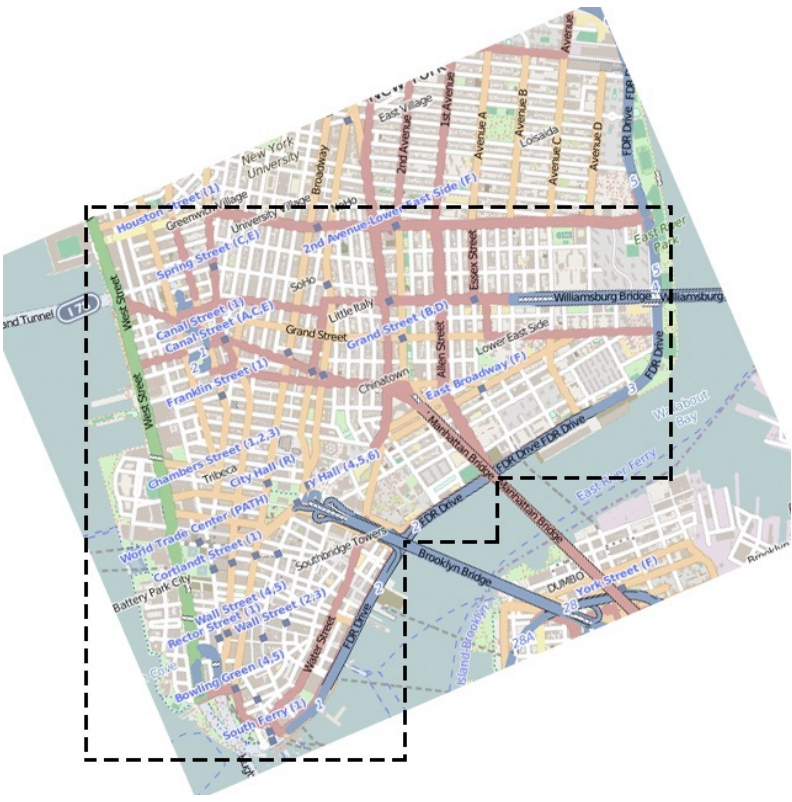

Figure 6: Lower Manhattan map in New York City

metric (i.e., CV) was chosen as it is a dimensionless number. However, after extensive testing, it proved to be of little benefit for our simulation data. We, therefore, neglect this measure within the context of this experiment. Likewise, the measured TL in our scheme was almost similar to that achieved by the baseline scheme, therefore we will omit these results as well.

As shown in Figure 7, the highest reduction of ATT in the Los Angeles scenario was 38\% decrease in travel time at the highest simulated density of vehicles. This demonstrates how our scheme would perform during on-peak travelling hours where the road network is overloaded by the vehicles. There was also a significant decrease in ATT with our scheme under an increasing density of vehicles compared to the baseline scheme (see Figure 7). This achieved improvement is almost proportional to the vehicles' density level, the higher the density the better improvement we get.

Similarly, Figure 8 shows how well our scheme performed when applied to NYC real world map. It can be seen also that the achieved reduction in ATT is proportional to the traffic load in the road network. In fact, our scheme performed better here than the Los Angeles scenario during light loads (i.e. off-peak times), with an almost $25 \%$ decrease in travel time. It is believed that this is because there tends to be more junctions and traffic light controllers in NYC. Furthermore, the efficiency of our scheme highly depends on the number of deployed TLCs from which the vehicles receive periodic information to assist them in optimizing their overall trip times. During on-peak times, however, the NYC scenario did not perform as well as the Los Angeles scenario in terms of the achieved ATT. Although this could be seen as a weakness of our scheme, we still believe that there are situations where stoppage cannot be avoided. This is the case in NYC where roads and streets are not large enough and commonly hold 


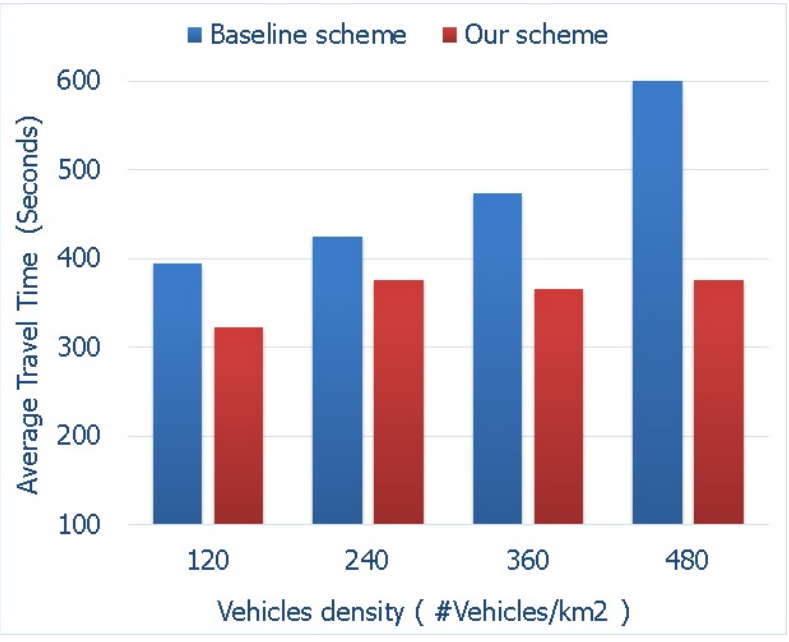

Figure 7: Average Travel Time in Los Angeles: our scheme vs. the baseline scheme

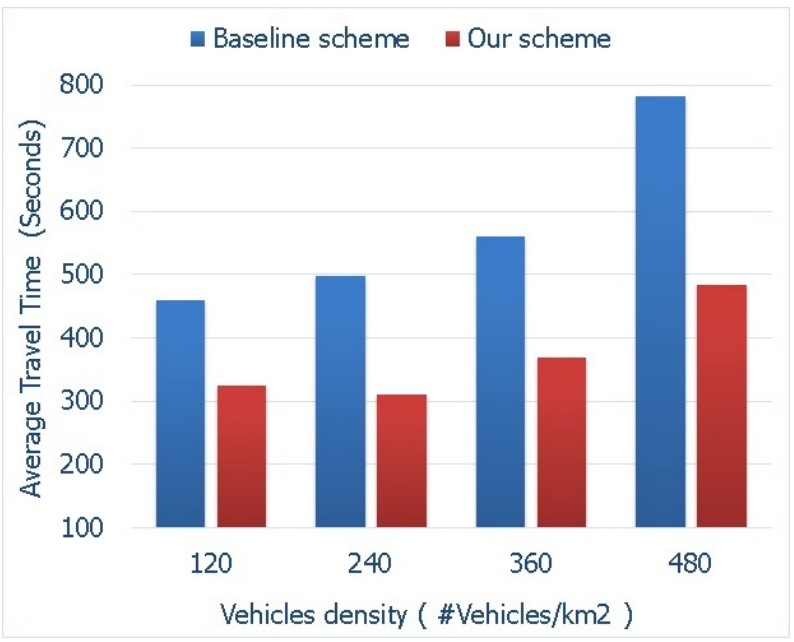

Figure 8: Average Travel Time in New York City: our scheme vs. the baseline scheme

high occupancy level on each road.

\section{CONCLUSION}

In this paper, we studied the traffic congestion problem that has been recognized as one of the major causes of air pollution, increased deaths on roads and considerable economic losses in the majority of fast growing cities worldwide. A V2I (Vehicle to Infrastructure) communication technologybased solution was, then, designed to alleviate the impact of such a challenging problem and ensure notable reduction of commuters' travel times. A key building block of this solution is the Belief-Desire-Intention architecture modelling the way a vehicle reasons and takes decisions based on its local knowledge as well as the knowledge acquired from the surrounding vehicles and the Traffic Light Controllers (TLCs) through the transmitted beacons. Following this architecture, each vehicle makes better informed and collaborative decisions about its speed (acceleration/deceleration) and position in the road segment (i.e. stay in the same lane or move to another one) in order to prevent stoppages at intersections whenever possible and accommodates the needs of its neighbors as long as it is safe to do so. The preliminary simulation results show interesting performance of the proposed solution and encourage us to do further investigation to extend and improve it. We will particularly extend our solution by implementing mechanisms to detect selfishly acting vehicles and collaboratively implement reactive and proactive solutions to encounter such behavior.

\section{ACKNOWLEDGEMENT}

Supported, in part, by Science Foundation Ireland grant 10/CE/I1855 and by Science Foundation Ireland grant 13/RC/2094.

\section{REFERENCES}

[1] D. Schrank, et al., TTIs 2012 Urban Mobility Report, Texas Transportation Institute, Texas A \& M University, 2012.

[2] National Highway Traffic Safety Administration, www.nhtsa.gov

[3] P. Savolainen and T. Datta, Evaluation of an Innovative Vehicle Alert System (EVAS), Report to Federal Highway Administration, Washington DC, 2007.

[4] S. Faye, et al., A Distributed Algorithm for Adaptive Traffic Lights Control, IEEE Conference on Intelligent Transportation Systems Anchorage, Alaska, USA, September 16-19, 2012.

[5] C.L.P. Chen, et al., A Real-Time Vehicle Navigation Algorithm in Sensor Network Environments, IEEE Transactions on ITS, Vol.13, No.4, pp.1657,1666, December 2012.

[6] Bell, Michael GH, et al., Time-dependent Hyperstar algorithm for robust vehicle navigation, Transportation Research Part A: Policy and Practice (2012).

[7] W. Min and L. Wynter, Real-time road traffic prediction with spatiotemporal correlations, Transportation Research Part C: Emerging Technologies 19(4), pp. 606-616, Elsevier, 2011.

[8] W. Shen and L. Wynter, Real-time road traffic fusion and prediction with GPS and fixed-sensor data, Information Fusion (FUSION), 15th International Conference on, Singapore, pp. 1468-1475, 2012.

[9] M. Collotta, et al., A Dynamic Traffic Light Management System Based on Wireless Sensor Networks for the Reduction of the Red-Light Running Phenomenon, Transport and Telecommunication, Vol. 15, No. 1, 2014.

[10] M. E. Bratman, Intentions, Plans, and Practical Reason, Harvard University Press: Cambridge, MA, 1987

[11] S. Djahel, et al., Adaptive Traffic Management for Secure and Efficient Emergency Services in Smart Cities, IEEE PerCom (WiP track), San Diego, California, USA, March 18-22, 2013.

[12] S. Djahel, et al., A Communications-oriented Perspective on Traffic Management Systems for Smart Cities: Challenges and Innovative Approaches, IEEE Communications Surveys and Tutorials, Vol. 17, No. $1,2015$.

[13] S. Wang, et al., A Multi-Agent Based Vehicles Re-routing System for Unexpected Traffic Congestion Avoidance, IEEE ITSC 2014, Oct. 8-11, 2014, Qingdao, China.

[14] R. A. Uzcategui and G. A. Marum, WAVE: A Tutorial, IEEE Communications Magazine, Vol. 47, No. 5, 2009.

[15] A. Wegener, et al., TraCI: an interface for coupling road traffic and network simulators, Proceedings of the 11th communications and networking simulation symposium, Ottawa, ON, Canada Apr. 13-16, 2008.

[16] M. Behrisch, et al., SUMO Simulation of Urban Mobility, Institute of Transportation Systems, German Aerospace Centre, 2011.

[17] S. Djahel and Y. Ghamri-Doudane, A Robust Congestion Control Scheme for Fast and Reliable Dissemination of Safety Messages in VANETs, IEEE WCNC 2012, Paris, France, pp. 2264-2269, April 1-4, 2012.

[18] H. I. Brahmi, et al., Messages Prioritization in IEEE 802.15.4 based WSNs for Roadside Infrastructure, IEEE ICCVE 2014, Nov. 3-7, 2014, Vienna, Austria. 